\title{
Catarata congénita bilateral: ambliopía por deprivación y su relación con el desarrollo
}

\section{Bilateral Congenital Cataract: Deprivation Amblyopia and its relation to development}

\author{
Lizbeth Uribe-Campos ${ }^{1,2 *}$, Leticia Arroyo-Muñoz ${ }^{3}$, Mario Mandujano-Valdés ${ }^{2}$, Hortensia Moreno-Macias ${ }^{4}$, \\ Juan C. Zenteno-Ruíz ${ }^{5,6}$, Roxana Muñoz-Hernández y Carmen Sánchez ${ }^{2}$
}

${ }^{1}$ Doctorado en Ciencias Biológicas y de la Salud, Universidad Autónoma Metropolitana; ${ }^{2}$ Laboratorio del Seguimiento del Neurodesarrollo del Instituto Nacional de Pediatría, Universidad Autónoma Metropolitana-Xochimilco; ${ }^{3}$ Fundación Hospital de Nuestra Señora de la Luz; ${ }^{4}$ Departamento de Economía, Universidad Autónoma Metropolitana-Iztapalapa; ${ }^{5}$ Departamento de Genética-Unidad de Investigación, Instituto de Oftalmología Conde de Valenciana; ${ }^{6}$ Departamento de Bioquímica, Facultad de Medicina, Universidad Nacional Autónoma de México; ${ }^{7}$ Departamento de Producción Económica, Universidad Autónoma Metropolitana-Xochimilco. México

\section{Resumen}

Objetivo: Determinar el impacto de la edad de cirugía en la catarata congénita bilateral (CCB) sobre los cambios en el desarrollo cognitivo, motor y del lenguaje, considerando los resultados de la función visual. Método: Se trata de un estudio descriptivo, longitudinal y ambispectivo de la evolución de la agudeza visual y del desarrollo, que utilizó las cartillas de agudeza visual de Teller, retinoscopia y la Escala de Desarrollo Infantil Bayley III. Fueron incluidos ocho pacientes con CCB para establecer las condiciones generales de inicio, con un seguimiento longitudinal después de la cirugía. Los pacientes fueron divididos en dos grupos según la edad de tratamiento (tratamiento temprano $<6$ meses y tratamiento tardío $>6$ meses). Se utilizó estadística no paramétrica mediante la prueba de tendencia a través de grupos ordenados utilizando un nivel de significancia de $p$ $<0.05$. A través de un análisis cualitativo se realizó una descripción del desarrollo visual. Resultados: El error refractivo disminuyó de manera progresiva y mostró una diferencia estadísticamente significativa entre ambos grupos $(p<0.01)$. El área cognitiva mostró mejores resultados en el grupo de tratamiento temprano $(p=0.012)$. Conclusión: El retraso en el tratamiento quirúrgico conduce a graves alteraciones en la organización de la función visual, así como en los comportamientos observables en el área motora, cognitiva y del lenguaje, lo que limita el desarrollo integral y adecuado en el individuo. Es necesaria la implementación de estrategias que incluyan la rehabilitación visual y la intervención temprana de alteraciones del desarrollo.

Palabras clave: Catarata congénita. Deprivación visual. Ambliopía. Función visual. Desarrollo.

\section{Abstract}

Purpose: To determine the impact of the age of surgery on bilateral congenital cataract (CCB), on changes in cognitive, language and motor development, considering the results of visual function. Methods: This is an ambispective descriptive longitudinal study of the evolution of visual acuity and development, using Teller Acuity Cards, Retinoscopy, and Bayley Scales of Infant and Toddler Development-Third edition. Eight patients with CCB were included to establish the general conditions

\section{Correspondencia:}

*Lizbeth Uribe-Campos

Alumnos, 53

Col. S. Miguel Chapultepec,

Del. Miguel Hidalgo

Fecha de recepción: 11-07-2017

Fecha de aceptación: 01-03-2018 E-mail: lizbethuribe_opt@ hotmail.com DOI: 10.24875/RMO.M18000030 www.rmo.com.mx
0187-4519/@ 2018 Sociedad Mexicana de Oftalmología. Publicado por Permanyer México. Este es un artículo Open Access bajo la licencia CC BY-NC-ND

Disponible en internet: 02-07-2018 Rev Mex Oftalmol. 2018;92(4):191-200 $\begin{array}{lll}\text { E-mail: lizbethuribe_opt @ hotmail.com } & \text { DOI: 10.24875/RMO.M18000030 www.rmo.com.mx } \\ \text { 0187-4519/@ } 2018 \text { Sociedad Mexicana de Oftalmología. Publicado por Permanyer México. Este es un artículo Open Access bajo la licencia CC BY-NC-ND }\end{array}$ (http://creativecommons.org/licenses/by-nc-nd/4.0/). 
of onset with a longitudinal follow-up after surgery. Patients were divided into two groups considering the age of treatment (early treatment $<6$ months; late treatment $>6$ months). We used non-parametric statistics by means of trend test in the two groups using a significance level of $p<0.05$. Through a qualitative analysis a description of the visual development was made. Results: Refractive errors decreased progressively with statistically significant difference between the two groups ( $p$ $<0.01)$. The cognitive area exhibited better outcomes with a significant difference $(p=0.012)$ in the early treatment group. Conclusions: Late surgical treatment led to severe changes in the organization of the visual function, as well as in observable behaviors of the motor, cognitive, and language areas, limiting the comprehensive and proper individuals' development. It is necessary to implement strategies that include visual rehabilitation and early intervention for development changes.

Key words: Congenital cataract. Visual deprivation. Amblyopia. Visual function. Development.

\section{Introducción}

A nivel mundial se estima que el número de niños con discapacidad visual es de 19 millones, de los cuales, 12 millones sufren errores de refracción que podrían ser fácilmente diagnosticables y corregibles ${ }^{1}$, más del $90 \%$ viven en países en desarrollo y más de las dos terceras partes se podrían haber evitado². Se estima que 1.4 millones de menores de 15 años sufren ceguera irreversible y necesitan rehabilitación visual para su pleno desarrollo psicológico y personal ${ }^{1}$. Las principales causas de ceguera en los niños varían de una región a otra y se encuentran asociadas en gran medida al desarrollo socioeconómico y a la disponibilidad de los servicios de atención médica ${ }^{3}$. Se estima que en casi la mitad de los niños ciegos se podría haber evitado la causa subyacente si se contara con un programa de detección visual ${ }^{3}$.

No obstante, hay causas importantes que son indistintas en todos los países, como las cataratas congénitas (CC), las anomalías oculares congénitas y las distrofias hereditarias de la retina.

La CC se refiere a una opacidad del cristalino adquirida durante el desarrollo prenatal ${ }^{4}$.Representa el $13 \%$ de las causas de disminución visual en niños y es considerada como la causa más frecuente de deprivación visual tratable. Tiene una prevalencia de 1 a 4 por cada 10,000 niños en países desarrollados y de 5 a 15 en países en vías de desarrollo ${ }^{4-8}$,con un reporte a nivel mundial de 200 mil niños ciegos por $\mathrm{CC}^{2,3,5,9}$.Es la causa más importante de ceguera y es responsable del 5 al $20 \%$ de cegueras en niños a nivel mundial ${ }^{9}$. En una revisión sistemática sobre la prevalencia de CC en varios países, se identificó una prevalencia promedio de 1.03 por cada 10,000 niños, con una incidencia promedio de 1.8 a 3.6 por cada 10,000 niños al año ${ }^{10}$.

En América Latina se estima que 1 de cada 300 nacimientos por año presentan CC, y es responsable del $20 \%$ de las causas de ceguera en la infancia ${ }^{11}$. En México, en una recopilación de datos provenientes de diferentes hospitales oftalmológicos de la Ciudad de México y del Instituto Nacional de Pediatría se aprecia que en los últimos 10 años se han realizado 260 diagnósticos de CC antes de los dos años de edad, de los cuales solo el $18.46 \%$ han sido sometidos a cirugía ${ }^{12-15}$. En el Hospital General de México se reporta que la CC de tipo bilateral (CCB) es más frecuente $(65 \%)$ que la de tipo unilateral (35\%), donde el estrabismo se presenta como la secuela más frecuente después de la cirugía $(25 \%)^{11}$. La principal causa de CC es idiopática, seguida de la heredada; sin embargo, puede deberse a una alteración metabólica (galactosemia, anemia hemolítica e hipoglucemia), o incluso tener un origen infeccioso cuando se presenta durante el primer trimestre del embarazo (síndrome de TORCH). No obstante, existen conjuntamente una gran variedad de síndromes cromosómicos y dismórficos con alto riesgo de desarrollarla (trisomía 21 y el síndrome de Turner) ${ }^{4,16}$.

El manejo de la CC va a depender de la etiología, lateralidad, tipo de catarata y del grado en que interfiere con la visión ${ }^{5}$. La decisión de operar dependerá del juicio clínico del oftalmólogo, que tendrá en cuenta la presencia de una privación visual importante o si la catarata es visualmente significativa, lo que dependerá de su morfología, tamaño, posición y densidad ${ }^{4}$. En la mayoría de los casos, el diagnóstico suele hacerse de manera tardía debido a que no se cuenta con un modelo único y universal para la evaluación del desarrollo visual en el niño; sin embargo, existen pruebas que determinan la integridad de la función visual, como la observación del patrón de fijación y el seguimiento visual ${ }^{17}$.

La ambliopía por deprivación puede definirse como una interrupción del desarrollo de la agudeza visual causada por una falta o inapropiada estimulación visual. Los periodos críticos y sensibles tienen un papel importante en el desarrollo visual ${ }^{18}$.La ambliopía ocasionada por la $\mathrm{CC}^{16}$ se debe a una anormalidad estructural anatómica y fisiológica ${ }^{19}$, que se acompaña de pérdida visual ${ }^{20}$. La reversibilidad de la ambliopía dependerá de los estados de maduración del sistema visual ${ }^{18}$, de la duración de la restricción y de la edad de tratamiento ${ }^{21}$.Si la ambliopía está presente durante 
el periodo crítico del desarrollo visual, las deficiencias ópticas y oculomotoras producirán la pérdida de conexiones neurales implicadas en la vía visual, que afectarán la visión binocular, que influye en el control y manipulación de tareas que requieren de la visión en tercera dimensión. Afectará, principalmente, la integración visual motora de tareas que requieren la percepción a profundidad y motricidad fina y que requieren la coordinación ojo-mano y ojo-pie ${ }^{22}$.

La detección y tratamiento temprano en pacientes con CC son importantes debido a que el desarrollo del niño en sus dos primeros años de vida está ligado fundamentalmente al movimiento y a la percepción. Los esquemas cognitivos relacionados con la construcción de la permanencia del objeto, la percepción del espacio y la imitación gestual pueden verse retrasados por dificultades visuales en la realización de movimientos que limitan el alcance, manipulación y exploración de objetos ${ }^{22}$.

Algunos estudios han establecido que los niños con deficiencia visual presentan un retraso en el desenvolvimiento neuropsicomotor por una limitada interacción con su ambiente y con objetos que favorecen el aprendizaje $^{23}$. El desarrollo de las funciones sensoriales necesita de la experiencia obtenida durante la infancia temprana, por lo que una anormalidad congénita puede modificar las habilidades visuales, auditivas y táctiles, lo que perturba la interacción con el ambiente ${ }^{24,25}$. Por lo tanto, la importancia del estudio radica en determinar el impacto del restablecimiento de la función visual en el área cognitiva, motora y del lenguaje para implementar estrategias de intervención específicas en la CC.

\section{Material y método \\ Diseño del estudio}

Se trata de un estudio observacional, descriptivo, longitudinal y ambispectivo ${ }^{26}$ para identificar el efecto de la edad de tratamiento en el restablecimiento de la función visual después de la cirugía de CC y del desarrollo de un programa de seguimiento. En todos los casos, los padres firmaron la carta de consentimiento informado y el protocolo se aprobó por los comités de ética e investigación del Instituto Nacional de Pediatría con el número de registro 047/2015.

\section{Población de estudio}

La población de estudio fueron menores de dos años de edad con diagnóstico de CCB, captados en el Departamento de Segmento Anterior de la Fundación
Hospital Nuestra Señora de la Luz, IAP, durante el periodo de octubre de 2012 a septiembre de 2014 y operados mediante la técnica quirúrgica de facoemulsificación. Fueron excluidos los niños que no asistieron a la evaluación de desarrollo antes de la cirugía y aquellos en que el especialista determinó situaciones oftalmológicas agregadas que afectaban el seguimiento. Fueron eliminados dos niños que presentaron complicaciones posquirúrgicas asociadas a una disminución importante de la visión o que fueron diagnosticados con alteraciones sistémicas que hacen esperar un retraso en el desarrollo. La investigación se realizó en ef Laboratorio de Seguimiento del Neurodesarrollo del Instituto Nacional de Pediatría de la Ciudad de México.

\section{Procedimiento}

Para evaluar la agudeza visual se utilizaron las cartillas de Agudeza Visual de Teller, para el estado refractivo se utilizó la retinoscopia, y para determinar el desarrollo cognitivo, motor y del lenguaje, la Escala de Desarrollo Infantil de Bayley-III. Se evaluó el valor refractivo en cada evaluación y se realizaron los cambios necesarios a la prescripción. Mediante la observación del examen clínico, se seleccionaron de la escala de Bayley-III las conductas que requieren la función visual para su desarrollo y expresión.

El programa de rehabilitación visual incluyó el uso de lentes de contacto después de la cirugía hasta el año de edad; en el segundo año se implementó el uso de lentes de armazón de visión sencilla con prisma para corregir la desviación ocular, y a partir de los dos años se modificó a uso de lentes bifocales para compensar la visión lejana y cercana.

El programa de intervención consistió en estrategias para favorecer el desarrollo cognitivo, motor (fino/grueso), del lenguaje (expresivo/receptivo) y personal so$\mathrm{cial}^{27}$. Se utilizaron estrategias para beneficiar el desarrollo de la función visual que involucraron tareas de fijación, seguimiento visual, coordinación oculomotora, manipulación gruesa y fina y coordinación ojo-mano/ ojo-pie. Se evaluaron la asistencia y la participación en el programa de intervención y la utilización de los lentes prescritos. Se realizó una evaluación preoperatoria basada en las condiciones iniciales y se aplicaron bimestralmente hasta la edad de 42 meses.

\section{Análisis de los datos}

Los pacientes fueron divididos en dos grupos de acuerdo a la edad de cirugía: tratamiento temprano 
( $<6$ meses) y tratamiento tardío ( $>6$ meses). En cada grupo se calculó la mediana de cada prueba a la que fueron sometidos, y se calculó la tendencia a través de una prueba no paramétrica para grupos ordenados, que es una extensión de la prueba de rangos de Wilcoxon, utilizando un nivel de significancia de $p<0.05$. Para el análisis se empleó el software estadístico Stata $®$ (StataCorp 2013, Stata Statistical Software).

La estimación de la agudeza visual fue convertida a unidades logMAR y el error refractivo fue utilizado en equivalente esférico para su análisis.

En el análisis cualitativo, de acuerdo con la evaluación de las conductas seleccionadas que fomentan la función visual, se identificaron tres tipologías ${ }^{28:} 1$ ) buena evolución (el desarrollo alcanzó valores cercanos a la normalidad); 2) evolución regular (el desarrollo, aunque mejoró, solo fluctuó cerca del promedio), y 3) mala evolución (el desarrollo permaneció por debajo del promedio).

\section{Resultados}

Se incluyeron 8 casos ( 4 niñas y 4 niños), de los que se obtuvieron 118 evaluaciones (promedio de 14.75 evaluaciones por paciente), con un seguimiento hasta los 42.1 meses de edad. La edad promedio de la cirugía en el grupo de tratamiento temprano fue de 5.6 meses (intervalo 4.9-6.0 meses) y en el grupo de tratamiento tardío de 11.3 meses (intervalo de 9.0-14.1 meses). En la tabla 1 se presentan las variables demográficas de los casos.

Presentaron nistagmo 4 pacientes desde el inicio del seguimiento y 7 presentaron desviación ocular antes y después de la cirugía de CCB.

\section{Función visual}

En la figura 1 se observa la evolución de la agudeza visual de cada ojo por separado por grupos. La agudeza visual fue tomada durante todo el seguimiento con el uso de la corrección óptica. En el grupo de tratamiento temprano, la agudeza visual muestra una mayor ganancia a pesar de la presencia de estrabismo, que persiste después de la cirugía. En este grupo, al finalizar el seguimiento, los casos se clasificaron con ambliopía de leve a moderada, mientras que los de tratamiento tardío se clasificaron con ambliopía de moderada a grave.

La ganancia en cuanto agudeza visual en cada ojo por separado mostró mejores resultados en el ojo derecho tanto en el tratamiento temprano como en el

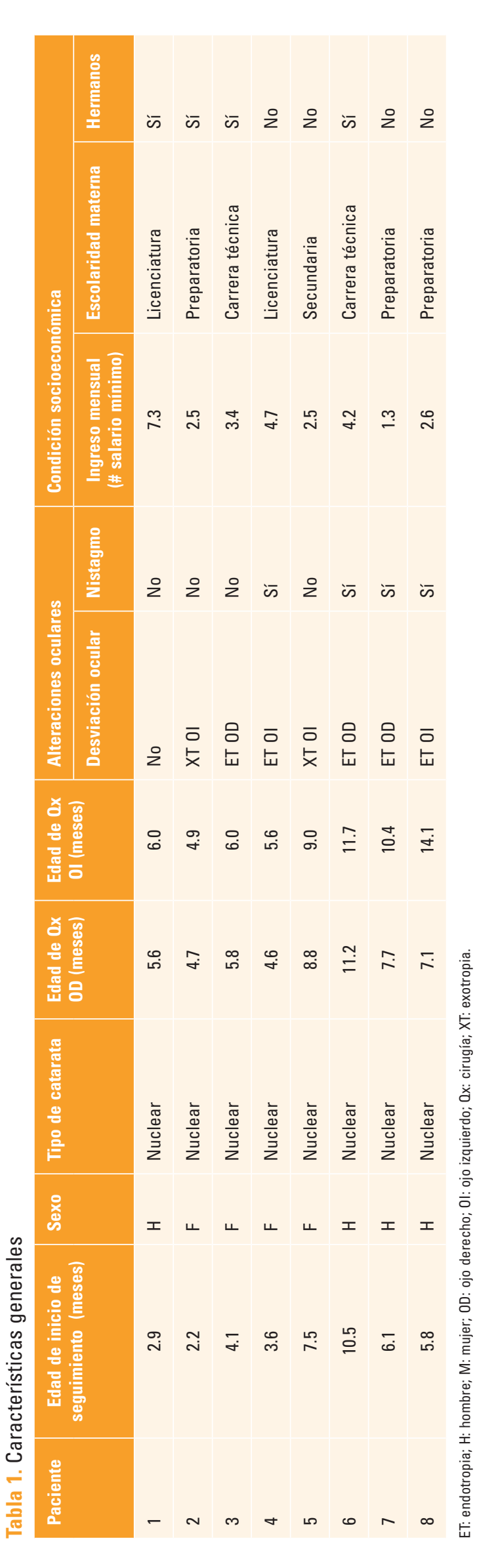



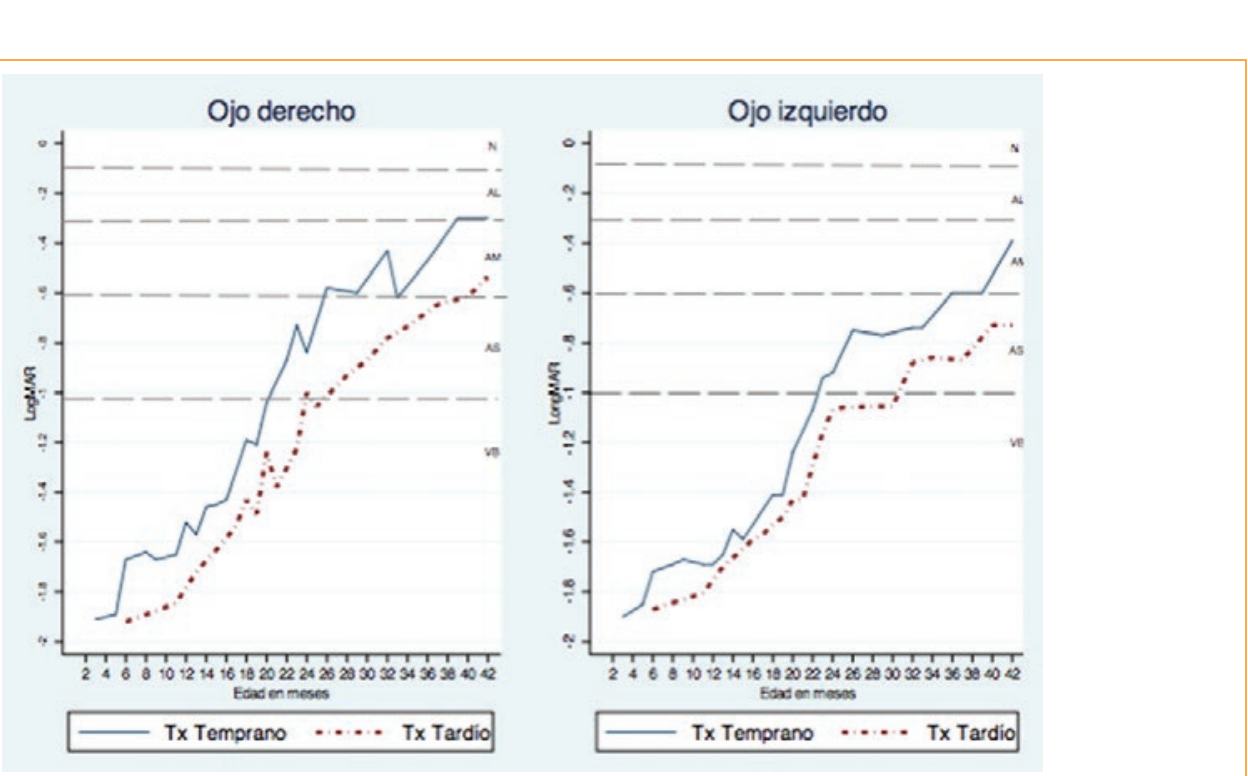

Figura 1. La agudeza visual posquirúrgica en el ojo derecho es ligeramente mejor que en el ojo izquierdo en ambos grupos con mejor desempeño en el grupo de tratamiento temprano. AL: ambliopía leve;

AM: ambliopía moderada; AS: ambliopía severa; N: normal; VB: visión baja.

tratamiento tardío (Fig. 1); sin embargo, la prueba estadística no mostró diferencia significativa $(p=0.459$ y $p=0.882$ ).

En ambos grupos, el valor refractivo disminuyó de manera progresiva y constante, con mayor disminución en el grupo de tratamiento temprano, que se inició con valores de alrededor de +5.00 dioptrías, que disminuyeron a - 0.50 dioptrías; en cambio, el grupo de tratamiento tardío se inició con valores cercanos a +10.00 dioptrías, que disminuyeron a +5.00 dioptrías (Fig. 2). La prueba estadística muestra una diferencia significativa entre ambos grupos para cada ojo $(p<0.01$ y $\mathrm{p}<0.01$ ).

\section{Desarrollo}

En todos los casos se observaron retrasos en todas las áreas antes de la cirugía. En el desarrollo cognitivo, los casos del grupo de tratamiento temprano alcanzaron un desarrollo clasificado como promedio desde los 13 meses. En cambio, los casos de tratamiento tardío evolucionaron por debajo del promedio, alcanzando valores por encima del promedio a los 31 meses (Fig. 3). La prueba estadística muestra resultados que reflejan una diferencia significativa entre ambos grupos $(p=0.012)$.

En cuanto al desarrollo del lenguaje, ambos grupos evolucionaron de una manera similar. El grupo de tratamiento temprano permaneció en un desarrollo por debajo del promedio hasta los 37 meses, momento en que alcanzó niveles promedio. Estos permanecieron hasta el final del seguimiento. Sin embargo, los casos de tratamiento tardío presentaron calificaciones inferiores hasta los 32 meses, momento en que alcanzaron calificaciones promedio (Fig. 4). No obstante, la prueba estadística no refleja una diferencia significativa entre ambos grupos $(p=0.677)$.

En el desarrollo motor, los casos de tratamiento temprano mostraron un desarrollo extremadamente bajo desde el periodo preoperatorio hasta los 12 meses después de la cirugía, momento en que alcanzaron calificación limítrofe y lograron niveles promedio hasta los 18 meses. Los de tratamiento tardío obtuvieron calificación limítrofe a los 4 meses, mejoraron hasta los 22 meses, cuando alcanzaron un valor promedio (Fig. 5). La prueba estadística no muestra diferencia significativa entre ambos grupos ( $p=0.398$ ).

Para la construcción de las tipologías se tomaron en cuenta las características generales tanto visuales como familiares (Tabla 2).

Se describió, además, el funcionamiento visual y su intervención en la expresión clínica y conductual durante el seguimiento en cuatro momentos: prequirúrgico, posquirúrgico, durante el seguimiento y en la última evaluación registrada (Tabla 3). 

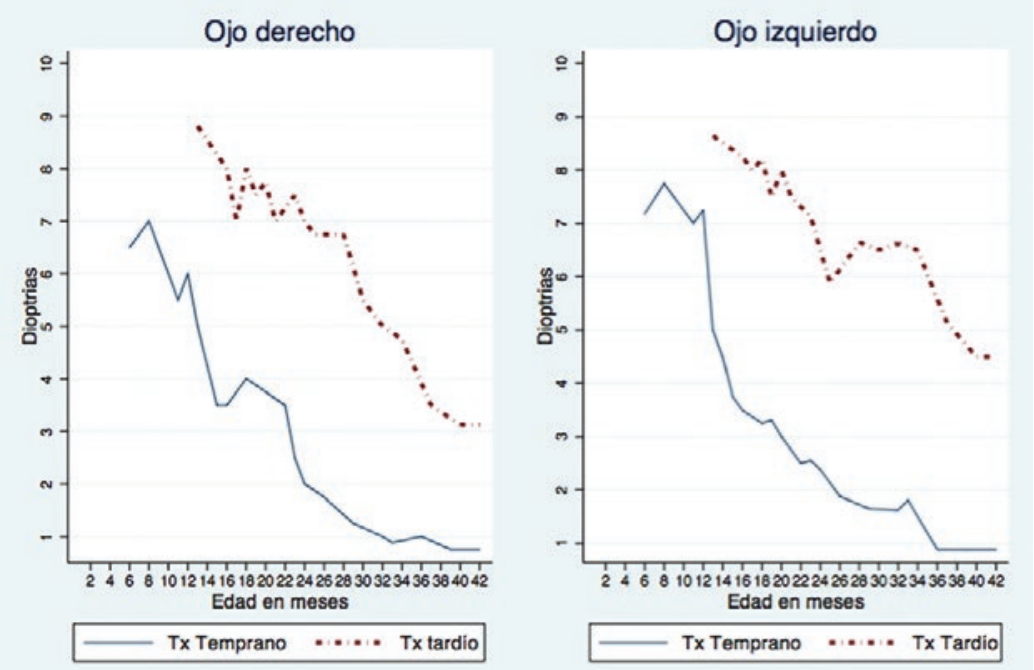

Figura 2. El error refractivo utilizando esférico equivalente es muy similar entre ambos ojos. El grupo de tratamiento temprano muestra valores de inicio menores, que mantiene durante todo el seguimiento Tx: tratamiento.

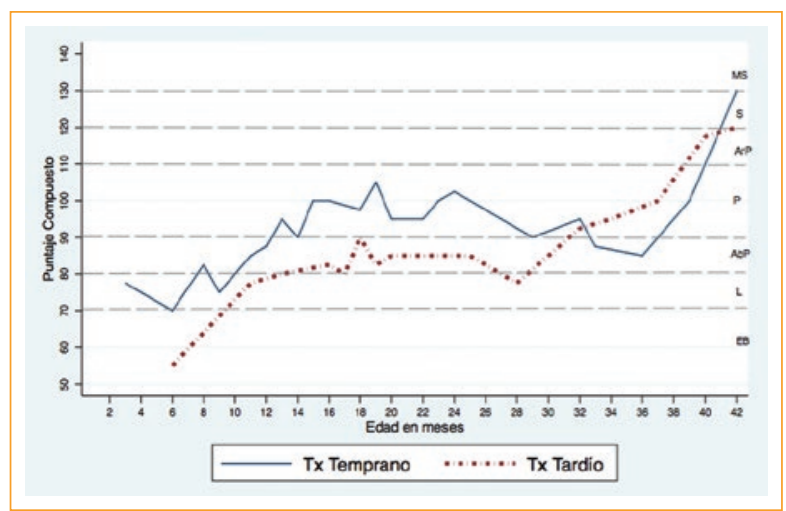

Figura 3. Escala de Desarrollo Infantil Bayley III. Escala cognitiva. El grupo de tratamiento temprano inicia con desarrollo limítrofe alcanzando valor promedio a partir de los 12 meses. El grupo de tratamiento tardío comienza con un desarrollo extremadamente bajo, no alcanzando un valor promedio hasta los 31 meses. AbP: abajo del promedio; ArP: arriba del promedio; EB: extremadamente bajo; L: limítrofe; MS: muy superior; P: promedio; S: superior; Tx: tratamiento.

Tomando en cuenta la tipología, los niveles alcanzados muestran que los niños con buena evolución logran valores esperados para la edad, principalmente al adquirir y mejorar las tareas visuales que involucran la fijación, precisión, coordinación ojo-mano y ojo-pie, a diferencia de los niños con una mala evolución que presentan dificultades en cada una de las áreas a pesar de la edad y las estrategias implementadas.

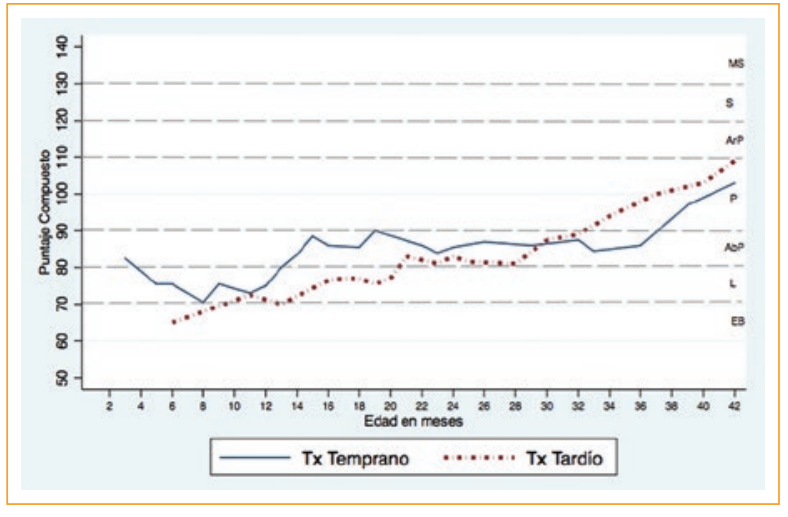

Figura 4. Escala de Desarrollo Infantil Bayley III. Escala de lenguaje. El grupo de tratamiento temprano logra un desarrollo promedio hasta los 37 meses, a diferencia del grupo de tratamiento tardío que lo alcanza desde los 32 meses. AbP: abajo del promedio; ArP: arriba del promedio; EB: extremadamente bajo;

L: limítrofe; MS: muy superior;

$\mathrm{P}$ : promedio; S: superior; Tx: tratamiento.

\section{Discusión}

La CC es considerada como una de las principales causas de disminución visual prevenible y se estima que casi la mitad de los niños con ceguera se podría haber evitado si se contara con la infraestructura adecuada en el servicio de salud, donde el diagnóstico y tratamiento temprano representan una ventana de oportunidad para el desarrollo de la función visual, así como de otras habilidades que permitan un óptimo desarrollo infantili3,29. 
Tabla 2. Tipología

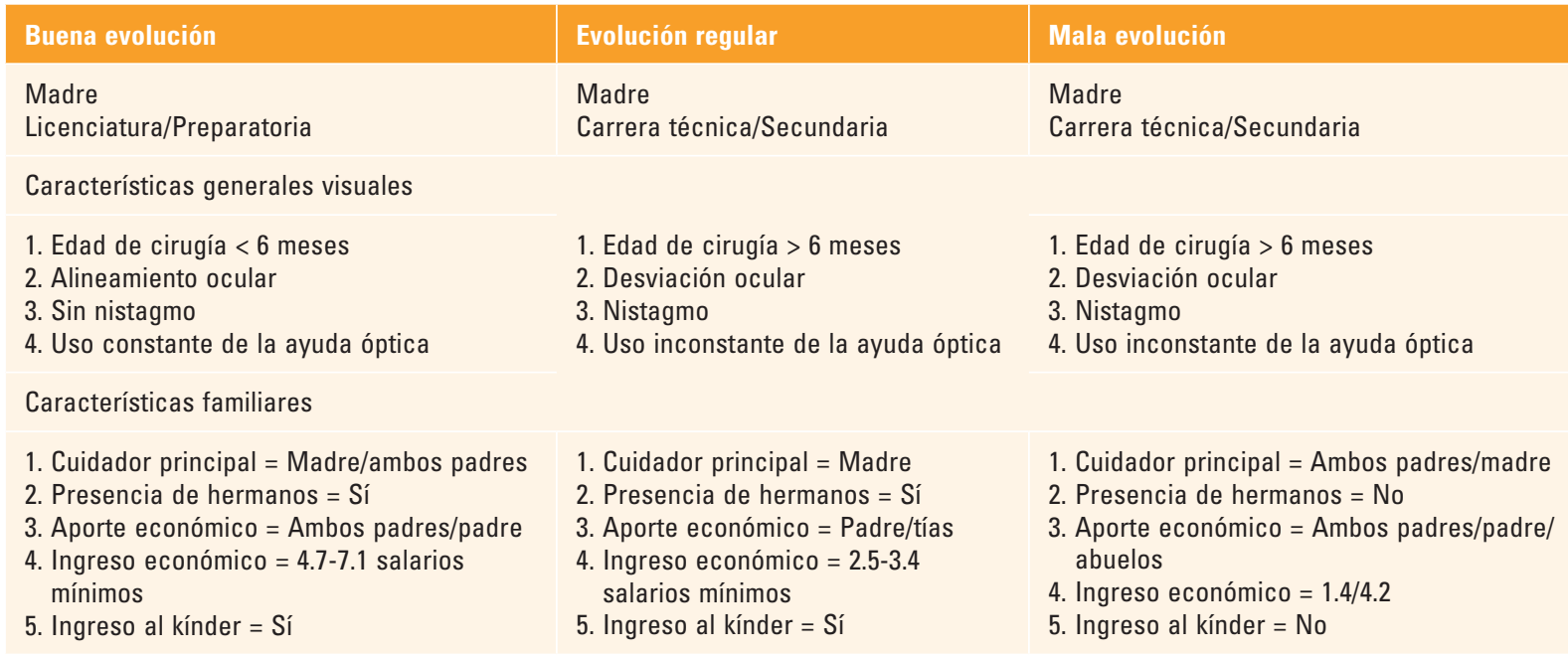

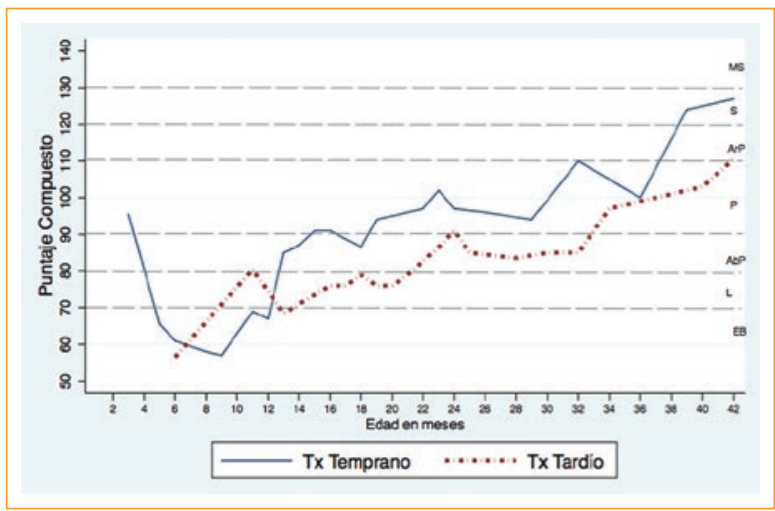

Figura 5. Escala de Desarrollo Infantil Bayley III. Escala motora. El grupo de tratamiento temprano alcanza un valor promedio desde los 19 meses, a diferencia del grupo de tratamiento tardío que lo consigue hasta los 33 meses. AbP: abajo del promedio;

ArP: arriba del promedio; EB: extremadamente bajo;

L: limítrofe; MS: muy superior; P: promedio;

S: superior; Tx: tratamiento.

Las evidencias actuales indican que la intervención quirúrgica debe realizarse en las primeras semanas de vida, ya que se considera un periodo caracterizado por cambios rápidos a nivel de la corteza visual ${ }^{30}$. Se ha establecido que la cirugía de la CCB debe llevarse a cabo dentro de las primeras 10 semanas, puesto que es considerado como un periodo sensible para la deprivación visual ${ }^{31}$; sin embargo, es necesario realizar un tratamiento posterior que incluya estrategias terapéuticas para evitar el estrabismo y la ambliopía ${ }^{32,33}$

En el caso de México el diagnóstico continúa realizándose de manera tardía a pesar de que la Secretaria de Salud resalta la importancia de la exploración visual en los primeros 6 meses de edad ${ }^{34}$. Tanto la inversión en el sistema de salud, ${ }^{35}$ como la falta de personal capacitado para realizar un tamiz visual en las primeras semanas de vida, podría afectar el resultado visual a largo plazo, puesto que la pérdida visual en los niños plantea estrategias particulares que son diferentes a la de los adultos ${ }^{35-37}$.

El estudio de la CC es relevante, dada su elevada frecuencia y la posibilidad de prevenir la ceguera mediante el tratamiento quirúrgico oportuno; sin embargo, la investigación sobre la evolución postoperatoria de la visión y el desarrollo cognitivo de los casos es un tema que ha sido escasamente estudiado. En México, al igual que en otros países de menor desarrollo, no se cuenta con estrategias y programas para la detección de anomalías visuales ni programas de rehabilitación visual temprana para este tipo de alteración ${ }^{38}$.

El interés de esta investigación radica en el análisis de las trayectorias del desarrollo durante un periodo prolongado de 42 meses, con numerosas evaluaciones de casos que no tuvieron la oportunidad de ser intervenidos quirúrgicamente en las primeras semanas de vida, cuya recuperación, tanto visual como cognitiva, aporta información relativa al periodo sensible de recuperación funcional y a las perspectivas de tratamiento tardío, problema frecuente en los países en desarrollo.

En el estudio, el tratamiento quirúrgico se realizó en concordancia con estándares internacionales, se colocó lente intraocular y no se presentaron complicaciones posquirúrgicas. Todos estuvieron incluidos en un programa de intervención y rehabilitación visual, y para la 
Tabla 3. Caracteristicas del funcionamiento visual

\begin{tabular}{|c|c|c|c|c|c|c|}
\hline \multicolumn{2}{|c|}{ Tipología } & \multirow{2}{*}{$\begin{array}{r}\text { Prequirúrgico } \\
\text { 1. Respuesta hacia la luz }\end{array}$} & \multirow{2}{*}{$\begin{array}{l}\text { Pos quirúrgico } \\
\\
\text { 1. Explora a su alre dedor } \\
\text { 2. Atención a objetos pequeños } \\
\text { 3. Sigue objetos en movimiento } \\
\text { 4. Akcanza y sujeta objetos } \\
\text { pequeños con barnido radial }\end{array}$} & \multirow{2}{*}{$\begin{array}{l}\text { Seguimiento } \\
\\
\text { on visual precisa } \\
\text { iniento visual horizortal y } \\
\text { coordinado } \\
\text { y saca objetos pequeños }\end{array}$} & \multicolumn{2}{|c|}{ Última evaluación } \\
\hline \multirow{2}{*}{$\begin{array}{c}\text { Buena } \\
\text { evolución }\end{array}$} & Adquirido & & & & $\begin{array}{l}\text { 1. Fijación visual precisa } \\
2 . \text { Seguimiento visual coordi } \\
\text { 3. Precisión en la manipulac } \\
\text { objetos pequeños } \\
\text { 4. Identifica formas y ubica c } \\
\text { 5. Ensarta cuentas }\end{array}$ & colores \\
\hline & $\begin{array}{l}\text { Con } \\
\text { alteración }\end{array}$ & $\begin{array}{l}\text { 2. Fijación visual débil hacia } \\
\text { obietos os } \\
\text { 3. Seguimiento visual horizontal } \\
\text { y vertical presente pero } \\
\text { limatado, con pérdidas de } \\
\text { fijadón }\end{array}$ & $\begin{array}{l}\text { 5. Seguimiento visual con mayor } \\
\text { precisión }\end{array}$ & $\begin{array}{l}\text { 4. Manipulación de objetos pequeños } \\
\text { con barrido radial } \\
\text { 5. Posición compensad ora de cabeza }\end{array}$ & & $\frac{1}{0}$ \\
\hline \multirow[b]{2}{*}{$\begin{array}{l}\text { Evolución } \\
\text { regular }\end{array}$} & Adquirido & 1. Respuesta hacia la luz & $\begin{array}{l}\text { 1. Explora a su alrededor } \\
\text { 2. Atención a objetos pequeños }\end{array}$ & & \multicolumn{2}{|c|}{$\begin{array}{l}\text { 1. Fijación visual con mayor predisión } \\
2 \text { Seguimiento visual coordinado } \\
\text { 3. Precisión en la manipulación de } \\
\text { objetos pequeños } \\
\text { 4. Identifica formas }\end{array}$} \\
\hline & $\begin{array}{l}\text { Con } \\
\text { alteración }\end{array}$ & $\begin{array}{l}\text { 2. Fijación débilhacia los } \\
\text { objetos } \\
\text { 3. Segumiento visual horizontal } \\
\text { y vertical lento y ímitado con } \\
\text { pérdidas de fijación } \\
4 \text {. Movimientos oculares hacia } \\
\text { arriba en busca de fijación }\end{array}$ & $\begin{array}{l}\text { 3. Seguimiento visual con mayor } \\
\text { precisión } \\
\text { 4. Oficutad para alcanzar objetos } \\
\text { 5. No sigue objetos en movimiento }\end{array}$ & $\begin{array}{l}\text { 1. Fijación visual imprecisa } \\
\text { 2. Segimimiento visual horizortal y } \\
\text { vertical impreciso } \\
\text { 3. Manipulación de objetos pequeños } \\
\text { con barrido radial } \\
\text { 4. Imprecisión para meter y sacar } \\
\text { objettos pe queños } \\
\text { 5. Posición compensadora de cabeza } \\
\text { 6. Entrecierra los ojos al fijar }\end{array}$ & \multicolumn{2}{|c|}{$\begin{array}{l}\text { 5. Ensarta cuentas con dificutad } \\
\text { 6. Dificultad para relacionar colores } \\
\text { conformas } \\
\end{array}$} \\
\hline \multirow[b]{2}{*}{$\begin{array}{c}\text { Mala } \\
\text { evolución }\end{array}$} & Adquirido & 1. Respuesta hacia la luz & $\begin{array}{l}\text { 1. Explora a su alrededor } \\
\text { 2. Atención a objetos pequeños }\end{array}$ & & & $\frac{E}{\frac{\varepsilon}{U}}$ \\
\hline & $\begin{array}{l}\text { Con } \\
\text { alteración }\end{array}$ & $\begin{array}{l}\text { 2. Fijación débil hacia los } \\
\text { objettos } \\
\text { 3. Segumiento visual horizontal } \\
\text { y vertical lento y ýmitado con } \\
\text { pérdidas de fijación } \\
\text { 4. Movimiertos oculares hacia } \\
\text { arriba en busca de fijación }\end{array}$ & $\begin{array}{l}\text { 3. Seguimiento visual con mayor } \\
\text { precisión } \\
\text { 4. Oficulad para alcanzar objetos } \\
\text { 5. No sigue objotos en movimiento }\end{array}$ & $\begin{array}{l}\text { 1. Fiación visual imprecisa } \\
\text { 2. Sequimiento visual horizontal y } \\
\text { vertical impreciso } \\
\text { 3. Manipulación de objetos pequehios } \\
\text { con barrido radal } \\
\text { 4. Imprecisión para meter y sacar } \\
\text { objetos pequeños } \\
\text { 5. Posición compensadora de cabeza } \\
\text { 6. Se acerca a la mesa de exploración } \\
\text { para observar detales }\end{array}$ & \multicolumn{2}{|c|}{$\begin{array}{l}\text { 1. Fipación visual imprecisa } \\
\text { 2. Seguimiento visual coordinado c } \\
\text { compensaciones de cabeza } \\
\text { 3. Dficultad en la manipulacoón de } \\
\text { objetos pequeños } \\
\text { 4. Dficultad para relacionar colores } \\
\text { con formas } \\
\text { 5. Ensartar cuent as con dícultad }\end{array}$} \\
\hline
\end{tabular}

evaluación del desarrollo se emplearon escalas idóneas y métodos estadísticos indicados en muestras pequeñas. Se intentaron comparar los resultados de dos grupos en función del momento de la intervención quirúrgica: temprana (antes de los 6 meses de edad) versus tardía (después de los 6 meses de edad), con respecto a la edad normalizada de las pruebas, como testigo histórico.

Los resultados muestran que la función visual se va estructurando en función de la edad de tratamiento, puesto que cada paciente ha adquirido de manera distinta cada uno de los procesos. El funcionamiento visual antes de la cirugía se caracterizó por pérdidas de fijación, con dificultad en las tareas visualmente dirigidas, con uso de compensaciones propioceptivas que se beneficiaron una vez realizada la cirugía. Algunos autores refieren que, conforme transcurre la edad, la experiencia visual y sus cambios funcionales contribuyen no solo a la propia función sino que facilitan a su vez y en paralelo la expansión de estructuras cognitivas. La práctica permite hacer discriminaciones más precisas que facilitan la adquisición y permanencia de estructuras ligadas a los estímulos visuales ${ }^{36,37}$.

El proceso de rehabilitación empleado fue crucial para construir un desarrollo adecuado. Las tareas que involucraron promover el desarrollo en todas las áreas partiendo de las estrategias para favorecer la función visual permitieron a los niños adquirir y mejorar sus habilidades, a pesar de la condición biológica inicial, El apoyo de los padres y la persistencia en la rehabilitación permitió que los niños operados a una edad menor alcanzaran un mejor desempeño.

En la literatura se discuten los resultados de la cirugía en función de la edad de tratamiento; sin embargo, numerosos estudios resaltan la importancia de realizar el tratamiento a edades tempranas. Se estima que el $80 \%$ de los operados de CC en etapas tempranas alcanzan una agudeza visual de 20/60, contrario a un tratamiento tardío que refiere resultados de 20/200 o peor $^{39}$, por lo que es indispensable después de la cirugía realizar una corrección óptica adecuadā junto con una rehabilitación visual y seguimiento multidisciplinario para disminuir las complicaciones en cuanto al resultado visual ${ }^{6}$, tomando en cuenta al individuo, a la familia y a su entorno ${ }^{40}$. En el presente estudio, el apoyo terapéutico multidisciplinario de estrategias para favorecer las capacidades visuales, cognitivas, motoras y del lenguaje resultó ser crucial para alcanzar un mejor desempeño, independientemente de la edad de 
cirugía, y en algunos niños incluso permitió llegar a valores de normalidad tanto en la función visual como en las diferentes áreas de estudio.

Se ha reportado que los pacientes con CCB manifiestan un retraso en la coordinación ojo-mano y oído-mano y en la localización del sonido, lo que repercute cognitivamente en la construcción de la permanencia del objeto. Estas restricciones pueden tener impactos acumulativos en el desarrollo del control postural, la coordinación y la movilidad proactiva ${ }^{41}$, debido a que la visión se halla profundamente integrada con todo el sistema de acción del infante, que involucra la postura, coordinaciones manuales, e incluso la inteligencia y personalidad ${ }^{42}$.

En las últimas décadas se han publicado estudios que abordan la repercusión del daño visual sobre el desarrollo infantil en las esferas de adaptación social, comprensión sensorimotora, exploración del medio ambiente, comprensión verbal y lenguaje expresivo; sin embargo, son pocos los artículos que han continuado con la investigación en esa línea. La literatura especializada sobre la CC es abundante, pero el interés se centra principalmente en el resultado de la función visual $^{43,44}$.

Los retrasos observados durante el seguimiento son parte del proceso del desarrollo. Postulamos que la reorganización de conductas anteriores ante las nuevas demandas dan paso a conductas más complejas relacionadas estrechamente con la mejora en el funcionamiento visual, pero de adquisición más lenta.

El estudio tiene la limitación de incluir un número reducido de casos, aunque se investigó la totalidad de los intervenidos en el hospital, cuyo seguimiento abarcó un periodo prolongado.

Los resultados obtenidos sugieren que la edad de tratamiento continúa siendo fundamental en el pronóstico visual; sin embargo, es posible observar que también interviene en la conducta global, cuyo diagnóstico integrado apunta a la necesidad de establecer paralelamente estrategias de intervención temprana que, al involucrar la implementación de una evaluación sistematizada del funcionamiento visual ante demandas estandarizadas (pruebas de desarrollo), permitan la intervención oportuna e integral en los niños, incluyendo el tratamiento óptico en sus diferentes modalidades, la rehabilitación visual y la intervención temprana de los problemas y alteraciones motrices, cognitivas, lingüísticas y emocionales, lo que permitiría un desarrollo humano integral y adecuado en el individuo.

\section{Conclusiones}

Los retrasos del tratamiento quirúrgico conducen a graves alteraciones en la organización del funcionamiento visual y dejan secuelas como la presencia de estrabismo, nistagmo y ambliopía. El desarrollo global, medido por los comportamientos observables en el área motora, cognitiva y del lenguaje, también se ve afectado por la edad de intervención quirúrgica. Se sugiere además que la duración del periodo sensible para la recuperación de la función visual parece no ser tan limitado y estrecho como lo reporta la literatura, donde la intervención tiene serias influencias en el pronóstico final.

La edad de tratamiento sigue siendo fundamental en el resultado de la agudeza visual, pero el desarrollo global también sufre modificaciones: los niños operados a una edad mayor presentan un menor desempeño, que mejora gradualmente gracias a la implementación de estrategias de intervención que favorecen su desarrollo.

\section{Agradecimientos}

El presente estudio es parte de la tesis de Doctorado de Lizbeth Uribe Campos, Maestra en Rehabilitación Neurológica, perteneciente al programa de Doctorado en Ciencias Biológicas y de la Salud de la Universidad Autónoma Metropolitana, Unidad Xochimilco.

\section{Responsabilidades éticas}

Protección de personas y animales. Los autores declaran que para esta investigación no se han realizado experimentos en seres humanos ni en animales.

Confidencialidad de los datos. Los autores declaran que han seguido los protocolos de su centro de trabajo sobre la publicación de datos de pacientes.

Derecho a la privacidad y consentimiento informado. Los autores han obtenido el consentimiento informado de los pacientes y/o sujetos referidos en el artículo. Este documento obra en poder del aütor de correspondencia.

\section{Financiamiento}

La investigación fue realizada con apoyo de la Fundación Hospital Nuestra Señora de la Luz, IAP, y del Instituto Nacional de Pediatría. 


\section{Conflicto de intereses}

\section{Durante la investigación no existió conflicto de intereses.}

\section{Bibliografía}

1. World Health Organization (WHO). Visual impairment and blindness Geneva; 2014. Disponible en: http://www.who.int/mediacentre/factsheets/ fs282/es/. Último acceso: 7 de noviembre de 2016.

2. World Health Organization (WHO). Global Initiative for the Elimination of Avoidable Blindness. Geneva; 1998. (unpublished document WHO/ PBL/97.61). Último acceso 26 de octubre de 2016.

3. Gilbert C, Foster A. Childhood blindness in the context of VISION 2020 the right to sight. Bull World Health Organ. 2001;79:227-232.

4. Ramos Gómez EA, Rodríguez Masó S, Copello Noblet M, Linares GuerraM, Reselló Leyva A, Rodríguez Cabrera N. Catarata congénita y baja visión. Habilitación visual en un grupo de pacientes. Rev Haban Cienc Méd. 2011:10:61-76.

5. Kim KH, Ahn K, Chung ES, Chung TY. Clinical outcomes of surgical techniques in congenital cataracts. Kor J Ophthalmol. 2008;22:87-91.

6. Agervi P, Kugelberg U, Kugelberg M, Zetterström C. Refractive and visual outcome of paediatric cataract surgery in the Ukraine. Acta Ophthalmo Scand. 2006:84:674-8

7. Gogate P, Gilbert C, Zin A. Severe visual Impairment and blindness in infants: Causes and opportunities for control. Middle East Afr J Ophthalmol. 2011;18:109-14

8. Drummond BA, Tartarella MB. Cirugía da catarata infantil unilateral. Arq Bras Oftalmol. 2008;71:238-41.

9. Foster A, Gilbert C, Rahi J. Epidemiology of cataract in childhood: a global perspective. J Cataract Refract Surg. 1997; 23:601-4.

10. Sheeladevi S, Lawrenson J, Fielder A, Suttle C. Global prevalence of childhood cataract: a systematic review. Eye. 2016;1-10.

11. Pérez Pérez JF, Arroyo Yllanes ME, Murillo Murillo L. Manejo de la catarata congénita: experiencia en el Hospital General de México. Rev Mex Oftalmol. 2005;79:139-44

12. Estadistics books (data base online). General Hospital of Mexico; 20052015. Actualizado el 26 de octubre de 2015.

13. Estadistics books. Association to Avoid Blindness, I.A.P; 2005-2015. Actualizado el 31 de octubre de 2015.

14. Estadistics books. National Institute of Pediatrics; 2005-2015. Actualizado el 5 de noviembre de 2015 .

15. Estadistics books. Nuestra Señora de la Luz IAP Foundation Hospital for the support provided; 2005-2015. Actualizado el 7 de noviembre de 2015.

16. Ejzenbaum F, Salomão SR, Berezovsky A, Waiswol M, TartareIla MB, Sacai PY, et al. Amblyopia after unilateral infantile cataract extraction after six weeks of age. Arq Bras Oftalmol. 2009;72:645-9.

17. Bustos ZM, Ortega RC, De la Fuente TM, Aguilar MG, Brechtel M, Hernández T, et al. Catarata congénita. Rev Hosp M Gea Glz. 2001;4:57-60.

18. Birch EE, Shimojo S, Held R. Preferential-looking assessment of fusion and stereopsis in infants aged 1-6 months. Invest Ophthalmol Vis Sci. 1985;26:366-70

19. Hensch TK. Critical period plasticity in local cortical circuits. Nat Rev Neurosci. 2005:6:877-88.

20. Mitchell DE, Sengpiel F. Neural mechanisms of recovery following early visual deprivation. Philos Trans R Soc B Biol Sci. 2009;364:383-98.
21. Magnusson G, Persson U. Screening for congenital cataracts: a cost-consequence analysis of eye examination at maternity wards in comparison to well-baby clinics. Acta Paediatr. 2005;94:1089-95

22. Webber AL, Wood JM, Gole GA, Brown B. The effect of amblyopia on fine motor skills in children. Invest Ophthalmol Vis Scie. 2008;49:594-603.

23. Araujo S, Estevam S, Bestillero L, Prado S. Description of the neuropsychomotor and visual development of visually impaired children. Arq Bras Oftalmol. 2010:73:526-30.

24. Banks MS, Salapatek P. Acuity and contrast sensitivity in $1-, 2-$, and 3-month-old human infants. Invest Ophthalmol Vis Sci. 1978;17:361-5.

25. Putzar L, Hötting K, Röder B. Early visual deprivation affects the development of face recognition and of audio-visual speech perception. Restor Neurol and Neurosci. 2010;28:251-7

26. Hernández SR, Fernández-Collado C, Baptista LP. Concepcion o elección del diseño de investigación. En: Metodología de la Investigación. 4th ed. México; 2006:157-234.

27. Sanchéz PC, Mandujano VM, Rivera GR, et al. El desarrollo integral del niño en el ciclo de vida y su cuidado. Ciencias Clínicas. 2008:2:43-51.

28. Taylor SJ, Bogdan R. Introduction to qualitative research methods. (Introducción a los métodos cualitativos de investigación). 2nd ed. Argentina: Paidos; 1987.

29. Gogate $P$, Kalua $K$, Courtright $P$. Blindness in Childhood in Developing Countries: Time for a Reassessment?. PLoS Med. 2009;6(12):e1000177.

30. Katsumi O, Chedid SG, Kronheim JK, Henry RK, Jones CM, Hirose T. Visual Ability Score - A new method to analyze ability in visually impaired children. Acta Ophthalmol. Scand. 1998;76:50-55.

31. Lloyd IC, Ashworth J, Biswas S, Abadi RV. Advances in the management of congenital and infantile cataract. Eye. 2007;21:1301-9.

32. Yorston D. Surgery for congenital cataract. Community Eye Health 2004; $17: 23-5$

33. Chan WH, Biswas S, Ashworth JL, Lloyd IC. Congenital and-infantile cataract: aetiology and management. Eur J Pediatr. 2012;171:625-30.

34. Instituto Méxicano de la Segurida Social (IMSS). Guía Práctica Clínica, Tratamiento Quirurgico de Catarata Congénita. Disponible en: http:// www.imss.gob.mx/sites/all/statics/guiasclinicas/620GRR.pdf. Ûltimo acceso 7 de octubre de 2016.

35. Nigenda G. El Seguro Popular de Salud en México: Desarrollo y retos para el futuro. En: Nota Técnica de Salud No. 2/2005.

36. Gardon GH, Gimenes GV, Pinheiro LM. A method to evaluate visual ability in infants. Arq Neuropsiquiatr. 2004;62:300-6.

37. Moss HA, Robson KS. The relation between the amount of time infants spend at various states and the development of visual behavior. Child Development. 1970;41:509-17.

38. Juárez-Muñoz IE, Rodríguez-Godoy ME, Guadarrama-Sotelo ME, Guerrero Anaya M, Mejía-Arangúre JM, Sciandra-Rico M. Incidence of common ophthalmological disorders in preschool children in Mexico City. Salud Pública de México. 1996;38:212-6.

39. Limburg H, Silva JC, Foster A. Cataract in Latin America: findings from nine recent surveys. Rev Panam Salud Publica. 2009;25:449-55.

40. Zetterström C, Kugelberg M. Paediatric cataract surgery. Acta Ophthalmol. Scand. 2007;85:698-710.

41. Sandoval N. Seguimiento del Neurodesarrollo en niños menores de 24 meses con diagnóstico de catarata congénita bilateral: Estudio de Casos. México: Universidad Autónoma Metrópolitana; 2011.

42. Gesell A. Diagnóstico del desarrollo normal y anormal del niño. Evaluación y manejo del desarrollo neuropsicológico normal y anormal del niño pequeño y el preescolar. 3. ${ }^{a}$ ed. México; 2006.

43. McConachie HR, Moore V. Early expressive language of severely visually impaired children. Dev Med And Child Neurol. 1994;36:230-40.

44. Reynell J. Developmental patterns of visually handicapped children. Child Care Health Dev. 1978;4:291-303. 